\title{
Personalization of the medical treatment of solid tumours using patient-derived tumour explants (Review)
}

\author{
CHRISTOPHE LOUANDRE ${ }^{1}$, JÉROME DONNADIEU ${ }^{2}$, EMMA LACHAIER $^{3}$, \\ CYRIL PAGE $^{2}$, BRUNO CHAUFFERT ${ }^{3,4}$ and ANTOINE GALMICHE ${ }^{1,4}$ \\ Departments of ${ }^{1}$ Biochemistry, ${ }^{2}$ Head and Neck Surgery and ${ }^{3}$ Medical Oncology, CHU Amiens Sud, \\ Amiens; ${ }^{4}$ EA4666, Université de Picardie Jules Verne (UPJV), Amiens, France
}

Received November 23, 2015; Accepted December 27, 2015

DOI: 10.3892/ijo.2016.3345

\begin{abstract}
Improving the pre-clinical characterization of therapeutic approaches and developing new biological assays that will enable treatment personalization for individual patients are promising developments in oncology. Here we describe a new approach consisting of culturing human tumour explants. This approach involves the preparation of slices from freshlyobtained, surgically-resected material that can be maintained ex vivo for several days. Recent studies have provided proof of principle that this approach can be easily implemented in order to explore the mode of action of various anticancer drugs and the responses of 'real' tumours at the individual patient level. We present the practical aspects and highlight the versatility of this approach, which allows for the analysis of the susceptibility of any individual tumour to multiple anticancer drugs in parallel. We discuss its potential as a companion assay in the design of optimal clinical trials and as a guide for the prescription of medical treatment. We discuss which future clinical and biological studies are needed to validate the information gathered from cultured tumour explants, and to integrate this information with that gathered from other assays in order to optimize the medical treatment of cancer.
\end{abstract}

\section{Contents}

1. Introduction

2. Technical aspects of the culture of tumour explants

Correspondence to: Dr Antoine Galmiche, Laboratoire de Biochimie, Centre de Biologie Humaine (CBH), CHU Amiens Sud, Avenue Laennec, 80054 Amiens Cedex, France

E-mail: galmiche.antoine@chu-amiens.fr

Abbreviations: EGFR, epidermal growth factor receptor; HGFR, hepatocyte growth factor receptor; PDX, patient-derived xenografts; HCC, hepatocellular carcinoma; HPS, hematoxylin phloxin saffron

Key words: tumour explants, therapeutics, pre-clinical research, treatment personalization, companion assay
3. Studying the responses of human tumours to drugs and tumour-targeting procedures

4. Anticipating the individual sensitivity of solid tumours to medical treatments

5. Conclusion and perspectives

6. Note added in proofs

\section{Introduction}

The expanding knowledge on the molecular basis of oncogenesis gathered in recent years has revealed the striking individual heterogeneity of solid tumours. Every single tumour bears a unique load of genetic, epigenetic and biochemical alterations, some of which play a driver role in carcinogenesis and offer potential therapeutic targets $(1,2)$. Several drugs directed against the major oncogenic actors are already available and clinically-approved (2). For example, receptor tyrosine kinases, such as the epidermal growth factor receptor (EGFR) are the target of many chemical inhibitors and biotherapies with excellent in vitro inhibitory efficacy (3). In clinical practice, however, therapeutic targeting is limited by the fact that the current level of genomic analysis does not translate into clear information regarding tumour sensitivity to most drugs. New biological analyses that would help to fill the existing gap between the exploration of the genome of cancer cells and its pharmacological sensitivity are awaited. In this review, we discuss how short-term culture of human tumour explants could be of interest in this respect.

\section{Technical aspects of the culture of tumour explants}

Tumour explants maintained in short-term culture have been used in the past to analyse various types of solid tumours, including breast carcinoma (4-9), hepatocellular carcinoma (HCC) (10), head and neck carcinoma $(11,12)$, melanoma (13), lung, prostate, colon (14), stomach, or pancreas carcinomas (15) and glioblastoma (16). This approach was not only applied to primary tumours, but also in some cases to their associated metastases (15). In these situations, tumour samples are relatively rapidly processed (ideally, within $30 \mathrm{~min}$ ) after surgical resection, considering the potentially deleterious effects of cold ischemia on cancer cells (15) (Fig. 1). Following 
1

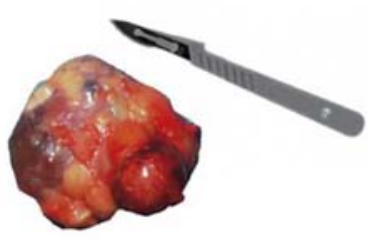

Surgical excision

3
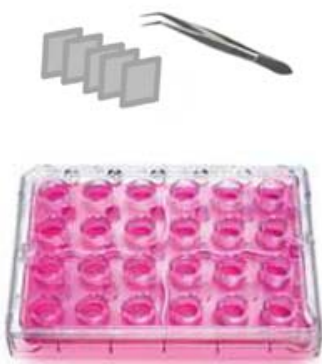

ex vivo culture for $24-96 \mathrm{~h}$
2

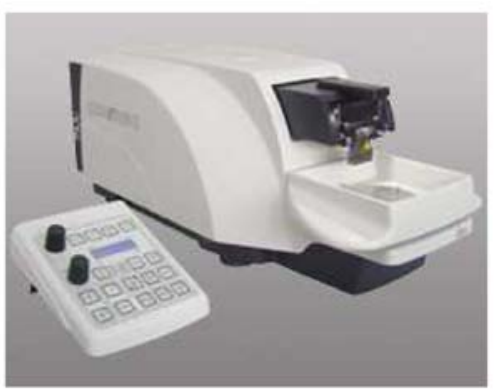

Preparation of slices

4

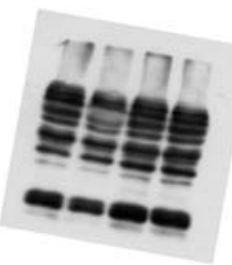

Biological analyses

Figure 1. A summary of the experimental steps carried out during the culture of tumour explants. Following surgical resection (1), the tumour sample is immersed in a saline solution and rapidly transferred to the pathology department, where it is prepared into slices of a calibrated thickness, using a microtome with a vibrating blade (2). Tumour explants are then transferred to a culture plate and can be exposed to various chemical compounds, monoclonal antibodies or therapeutic reagents (3). After a typical exposure time of $48 \mathrm{~h}$, tumour samples can be analyzed by immunohistochemistry, immunoblot analysis, or any molecular technique (4).
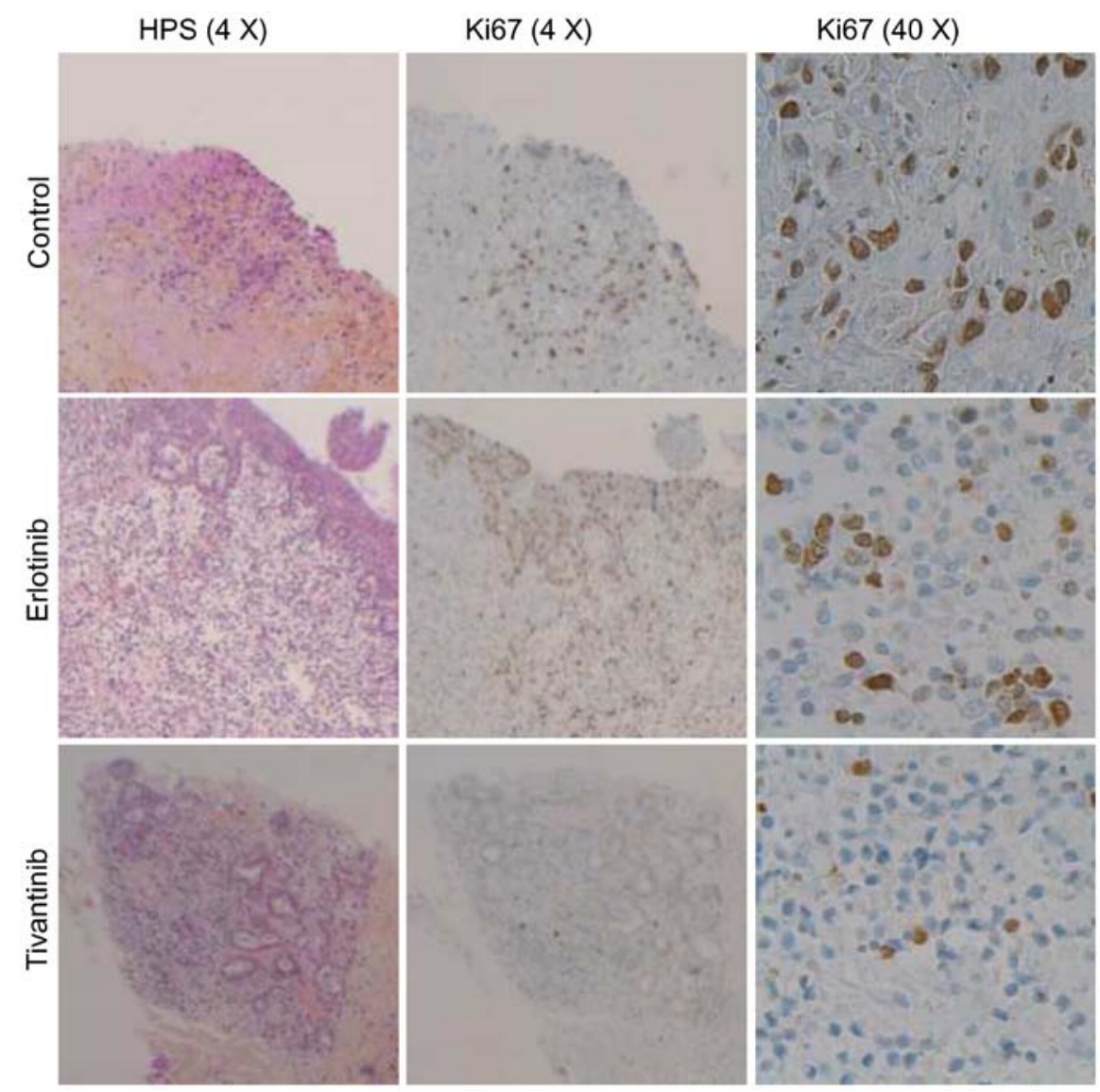

Figure 2. Immunohistological analysis of tumour cell proliferation in explants prepared from a head and neck squamous cell carcinoma tumour maintained in culture for $48 \mathrm{~h}$. The left panels are stained with hematoxylin phloxin saffron (HPS). Tumour cell proliferation was analyzed by performing Ki67 labelling (brown labelling, right panels) on tumour exaplants that had been maintained in culture for $48 \mathrm{~h}$ in the presence of erlotinib (1 $\mu \mathrm{M}$, an inhibitor of the EGFR) or tivantinib $[1 \mu \mathrm{M}$, an inhibitor of the hepatocyte growth factor receptor (HGFR)]. Note the preservation of tumour architecture and the presence of a strong contingent of Ki67-positive, proliferating tumour cells, in control conditions (magnification, $\mathrm{x} 4$ and $\mathrm{x} 40$ as indicated). 
macroscopic identification of viable regions from the resected material, tumour samples can be prepared by manual dissection (10). Although manual preparation of tumour samples is technically simple and easily implemented, it nevertheless presents the drawback of isolating fragments of different shapes and thicknesses, obtained from tumour regions of heterogeneous composition. Preparing tumour slices using a microtome equipped with a vibrating blade represents a significant improvement, since it results in slices with a standardized and reproducible thickness. In most studies to date, slices were prepared at a thickness of $<300 \mu \mathrm{m}$. This technical design limits the creation of artifactual hypoxic areas and facilitates uniform access of anticancer drugs to all cells in the tumour sample (11). Using this design, tumour samples can be maintained in conventional culture conditions for several days. In all studies published so far, tumour viability was preserved satisfactorily for $\geq 48$ hours, allowing for adequate exposure of the tumour cells to chemotherapeutic agents and targeted therapies (4-16).

A great advantage of culturing tumour slices over techniques that rely on the isolation of cancer cells is that the conditions are kept as similar to the clinical situation as possible. Compared to the use of cancer cell lines or even explanted cancer cells maintained in primary culture, the use of tumour slices permits the implementation of in vitro studies that take into account: i) the heterogeneous cellular composition of tumours. Non-tumour accessory cells, such as cancer-associated fibroblasts are present, as well as the multiple cellular lineages that constitute the tumour itself. ii) The complex 3D organization of solid tumours. Cancer cells are known to establish complex interactions with each other and also with the extracellular matrix, a parameter that can dramatically modulate their response to chemotherapeutic agents (17-19). This aspect of their physiology is preserved when tumour slices are prepared and used in short-term culture. The best evidence that ex vivo culture does not radically alter the physiology of the tumour explants comes from the observation that short-term culture of $48 \mathrm{~h}$ has little effect on the proliferation index of most solid tumours, as was for example shown in breast cancer (5) (Fig. 2).

After the culture step, all types of histological, biochemical and molecular analyses can be performed to measure tumour cell proliferation $(4,5,8,11)$, detect the occurrence of genomic lesions or cell death by apoptosis (11) or examine the activation levels of oncogenic signal transduction cascades $(7,10,14$; and unpublished data). Short-term culture of tumour explants is therefore a versatile approach that can be easily implemented in order to study the effects of most medical treatments on cancer cells in individual tumours.

\section{Studying the responses of human tumours to drugs and tumour-targeting procedures}

Cancer cell lines, grown as monolayers or xenografts in immunosuppressed animals, are widely used and have proved instrumental in validating the pre-clinical rationale for most targeted therapies and chemotherapeutic drugs available today in clinics (20). They are however known to produce artifacts (21), which could partially explain the failure of a high percentage of new candidate drugs entering the initial clinical evaluation after a promising pre-clinical characterization (22). This major bottleneck in anticancer drug discovery reflects the need for better and more clinically-relevant experimental systems to study tumour drug-sensitivity (22). New developments and experimental approaches, such as the use of multicellular tumour spheroids $(20,23)$ or the derivation of tumour organoids from surgical samples (24) might improve the accuracy of cellular models. Patient-derived xenografts (PDX), i.e., models based on the implantation of cancerous tissue from a patient's primary tumour into immunodeficient mice, are currently considered as the gold standard for the study of individual tumour sensitivity $(25,26)$. Tumours maintained as PDX bear most of the pathological characteristics of the tumour from which they originate (25). The use and the maintenance of PDX constitutes, however, a relatively heavy and costly procedure, restricted in practice to a limited number of laboratories. They are also not devoid of potential pitfalls and limitations (27). Firstly, not every tumour can be maintained as a PDX. Secondly, successive tumour passages as xenografts result in the emergence of cancer clones with adapted physiology $(27,28)$. It is therefore increasingly clear that using any single model, even PDX, is not sufficient for determining tumour sensitivity to different drugs.

The culture of tumour explants offers a useful alternative because it makes it possible to evaluate the response of a relatively large number of 'real' tumours at a much lower cost than with PDX. In addition, short-term culture allows for the study of tumour responses under defined conditions and in response to a broad array of drugs, irrespective of the pharmacological and toxicological considerations that are encountered in animal models. There are however theoretical limitations to the use of tumour explants, the most evident ones being the impossibility to study the longterm effects of medical treatments, and those that depend on the recruitment of immune cells from blood. Shortterm culture of tumour explants is therefore not suited to study the long-term consequences of vascular involution induced by anti-angiogenic treatments or immune checkpoint modulators. Rather, it is adapted for the analysis of medical compounds that act directly at the level of tumour cells, either to resume cell proliferation or induce cancer cell death. Importantly, the culture of tumour explants does not take into account the pharmacokinetic determinants of tumour sensitivity (29).

With these limitations of the culture of tumour explants in mind, we used this strategy to analyse the response of hepatocellular carcinoma (HCC) to sorafenib, the treatment of reference for advanced stages of this tumour (10). The results revealed the striking heterogeneity of the individual responses of HCC to sorafenib. Sorafenib efficiently reduced the activation levels of the oncogenic cascade RAF-MEK-ERK in two out of six HCC tumours (10). In some cases, sorafenib did not only fail to control this cascade, but eventually even paradoxically activated it (10). Recently, Gerlach et al also used this approach to analyse at the individual level the cytotoxic response induced by cisplatin and docetaxel, i.e., two chemotherapeutic agents, in head and neck squamous cell carcinoma (11). These studies and others indicate that culturing tumour explants can provide interesting information on fundamental 
aspects of the mode of action of anticancer drugs on tumours at the individual patient level.

\section{Anticipating the individual sensitivity of solid tumours to medical treatments}

In the clinical setting, the prescription of medical treatment often relies on a process of trial and error (29). Over the past decade, the introduction of tumour genotyping-based biomarkers into clinical practice has permitted substantial progress for a number of solid tumours $(1,2)$. The introduction of trastuzumab for the treatment of advanced breast cancer was the first clinical situation to illustrate this new concept of therapeutic prescription guided by the analysis of a tumour biomarker (in this case the immunohistochemical analysis of HER2 overexpression) (30). While such patient stratification based on genome analysis is certainly an optimal situation, it does not apply to most patients with solid tumours. In most cases, the current level of genomic analysis does not predict the individual tumour sensitivity $(31,32)$. This situation is not only a missed opportunity for most patients with solid tumours, but it also represents a major hindrance for the introduction of new anticancer drugs into clinical practice. Indeed, patient stratification based on biomarkers of drug response is essential for the design of successful clinical trials in oncology (29). A major aim of oncology research is now to validate new approaches to provide reliable information about individual tumour sensitivity, and thereby enable the design of clinical trials with a higher rate of success.

Strategies based on PDX and genetically-engineered mouse models are among the most promising for improving pre-clinical evaluation of therapeutic treatments and designing better clinical trials $(33,34)$. A discussion about the set-up of the so-called co-clinical trials, performed in patients and on tumour 'avatars' maintained in mice, is beyond the scope of the present review (35). Nevertheless, such studies pose several practical difficulties and require specialized structures ('mouse hospitals') providing an adapted framework for the implementation and the clinical integration of the results obtained in mice (35). Another major drawback of this approach is the long time that is required to establish tumour xenografts. This limitation of PDX will prevent most patients from benefiting from the information gained from their own tumour avatars (35).

Having in mind these limitations, we propose that a potential utility of tumour explants maintained in culture could lie in its clinical application as a companion assay for the prediction of individual tumour sensitivity to drugs. Tumour explants may be used during patient recruitment for clinical trials, as a means of enriching a target population, and also in order to estimate the number of patients that need to be recruited. In theory, slices could also be used whenever tumour material is accessible in order to expose tumour slices to a panel of drugs ex vivo and screen the most active molecules available. A further advantage of short-term culture of tumour explants over PDX is that this approach can deliver information more rapidly (in less than two weeks), i.e., in a time-frame that is compatible with the process of clinical decision making.

Unfortunately, no study to date has yet attempted to relate the information gathered from the culture of tumour explants with the clinical response of patients to anticancer drugs. This is certainly due to the fact that this approach requires surgical resection of the tumour material, and all studies to date have explored the use of slices after curative surgery (4-16). In order to establish the clinical relevance of culturing tumour slices, we propose that future studies could be centred on oligometastatic disease, i.e., the state of limited systemic dissemination that can be observed in several types of solid tumours (36). In this context, surgical access to a local metastasis or to the primary tumour itself might provide an opportunity to prepare tumour slices and explore a limited array of parameters (markers of apoptosis, proliferation, genomic alterations) in tumour explants exposed to a panel of anticancer drugs.

\section{Conclusion and Perspective}

Short-term culture of human tumour explants was recently applied to study the responses of different solid tumours to various therapeutic compounds. Because this approach holds the potential of improving the most fundamental aspects of our understanding of the mechanisms of action of drugs and also the design of future clinical trials, it could be instrumental in reducing the current gap between the biology laboratory and the clinic. Its use as a reliable readout of individual tumour sensitivity is also a promising perspective, but has not yet been established. Provided that such validation can be obtained in future clinical studies, the culture of tumour explants, together with other functional assays exploring specific aspects of tumour biology (37) could assist oncologists in the design of better and more successful clinical trials, and ultimately, in the personalization of patient treatment.

\section{Note added in proofs}

Following the submission of our manuscript, we became aware of a paper by Majumder et al (38), which for the first time reports the use of patient-derived tumour explants for predicting the clinical response of head and neck squamous cell carcinoma or metastatic colorectal cancer to anticancer drugs. This study constitutes an important validation of the utility of tumour explants for the personalization of medical treatment of tumours.

\section{Acknowledgements}

This study was supported by la Ligue contre le Cancer, comité de la Somme. We are grateful to Zuzana Saidak and Chloé Sauzay for critical reading of the manuscript.

\section{References}

1. Stratton MR: Exploring the genomes of cancer cells: Progress and promise. Science 331: 1553-1558, 2011.

2. Eifert $\mathrm{C}$ and Powers RS: From cancer genomes to oncogenic drivers, tumour dependencies and therapeutic targets. Nat Rev Cancer 12: 572-578, 2012

3. Davis MI, Hunt JP, Herrgard S, Ciceri P, Wodicka LM, Pallares G, Hocker M, Treiber DK and Zarrinkar PP: Comprehensive analysis of kinase inhibitor selectivity. Nat Biotechnol 29: 1046-1051, 2011 
4. van der Kuip H, Mürdter TE, Sonnenberg M, McClellan M, Gutzeit S, Gerteis A, Simon W, Fritz P and Aulitzky WE: Short term culture of breast cancer tissues to study the activity of the anticancer drug taxol in an intact tumor environment. BMC Cancer 6: 86, 2006.

5. Dean JL, McClendon AK, Hickey TE, Butler LM, Tilley WD, Witkiewicz AK and Knudsen ES: Therapeutic response to CDK4/6 inhibition in breast cancer defined by ex vivo analyses of human tumors. Cell Cycle 11: 2756-2761, 2012.

6. Séveno C, Loussouarn D, Bréchet S, Campone M, Juin P and Barillé-Nion S: $\gamma$-Secretase inhibition promotes cell death, Noxa upregulation, and sensitization to $\mathrm{BH} 3$ mimetic ABT-737 in human breast cancer cells. Breast Cancer Res 14: R96, 2012.

7. Grosso SH, Katayama ML, Roela RA, Nonogaki S, Soares FA, Brentani H, Lima L, Folgueira MA, Waitzberg AF, Pasini FS, et al: Breast cancer tissue slices as a model for evaluation of response to rapamycin. Cell Tissue Res 352: 671-684, 2013.

8. Holliday DL, Moss MA, Pollock S, Lane S, Shaaban AM, Millican-Slater R, Nash C, Hanby AM and Speirs V: The practicalities of using tissue slices as preclinical organotypic breast cancer models. J Clin Pathol 66: 253-255, 2013.

9. Pennington K, Chu QD, Curiel DT, Li BD and Mathis JM: The utility of a tissue slice model system to determine breast cancer infectivity by oncolytic adenoviruses. J Surg Res 163: 270-275, 2010.

10. Godin C, Dupont S, Ezzoukhry Z, Louandre C, Chatelain D, Henaut L, Sabbagh C, Regimbeau JM, Maziere JC, Barbare JC, et al: Heterogeneous sensitivity of hepatocellular carcinoma to sorafenib revealed by the short-term culture of tumor fragments. Anticancer Res 33: 1415-1420, 2013.

11. Gerlach MM, Merz F, Wichmann G, Kubick C, Wittekind C, Lordick F, Dietz A and Bechmann I: Slice cultures from head and neck squamous cell carcinoma: A novel test system for drug susceptibility and mechanisms of resistance. Br J Cancer 110 : 479-488, 2014.

12. Peria M, Donnadieu J, Racz C, Ikoli JF, Galmiche A, Chauffert B and Page C: Evaluation of individual sensitivity of head and neck squamous cell carcinoma to cetuximab by short-term culture of tumor slices. Head Neck: May 20, 2015 (Epub ahead of print). doi: 10.1002/hed.24126.

13. Micel LN, Tentler JJ, Tan AC, Selby HM, Brunkow KL, Robertson KM, Davis SL, Klauck PJ, Pitts TM, Gangolli E, et al: Antitumor activity of the MEK inhibitor TAK-733 against melanoma cell lines and patient-derived tumor explants. Mol Cancer Ther 14: 317-325, 2015.

14. Vaira V, Fedele G, Pyne S, Fasoli E, Zadra G, Bailey D, Snyder E, Faversani A, Coggi G, Flavin R, et al: Preclinical model of organotypic culture for pharmacodynamic profiling of human tumors. Proc Natl Acad Sci USA 107: 8352-8356, 2010.

15. Corben AD, Uddin MM, Crawford B, Farooq M, Modi S, Gerecitano J, Chiosis G and Alpaugh ML: Ex vivo treatment response of primary tumors and/or associated metastases for preclinical and clinical development of therapeutics. J Vis Exp 92: e52157, 2014

16. Merz F, Gaunitz F, Dehghani F, Renner C, Meixensberger J, Gutenberg A, Giese A, Schopow K, Hellwig C, Schäfer M, et al: Organotypic slice cultures of human glioblastoma reveal different susceptibilities to treatments. Neuro-oncol 15: 670-681, 2013.

17. Nakasone ES, Askautrud HA, Kees T, Park JH, Plaks V, Ewald AJ, Fein M, Rasch MG, Tan YX, Qiu J, et al: Imaging tumor-stroma interactions during chemotherapy reveals contributions of the microenvironment to resistance. Cancer Cell 21: 488-503, 2012

18. Correia AL and Bissell MJ: The tumor microenvironment is a dominant force in multidrug resistance. Drug Resist Updat 15: 39-49, 2012.

19. Gomes LR, Vessoni AT and Menck CF: Three-dimensional microenvironment confers enhanced sensitivity to doxorubicin by reducing p53-dependent induction of autophagy. Oncogene 34: 5329-5340, 2015.

20. Sharma SV, Haber DA and Settleman J: Cell line-based platforms to evaluate the therapeutic efficacy of candidate anticancer agents. Nat Rev Cancer 10: 241-253, 2010.
21. Daniel VC, Marchionni L, Hierman JS, Rhodes JT, Devereux WL, Rudin CM, Yung R, Parmigiani G, Dorsch M, Peacock CD, et al: A primary xenograft model of small-cell lung cancer reveals irreversible changes in gene expression imposed by culture in vitro. Cancer Res 69: 3364-3373, 2009.

22. Begley CG and Ellis LM: Drug development: Raise standards for preclinical cancer research. Nature 483: 531-533, 2012.

23. Lao Z, Kelly CJ, Yang XY, Jenkins WT, Toorens E, Ganguly T, Evans SM and Koch CJ: Improved methods to generate spheroid cultures from tumor cells, tumor cells \& fibroblasts or tumorFragments: Microenvironment, microvesicles and miRNA. PLoS One 10: e0133895, 2015.

24. van de Wetering M, Francies HE, Francis JM, Bounova G, Iorio F, Pronk A, van Houdt W, van Gorp J, Taylor-Weiner A, Kester L, et al: Prospective derivation of a living organoid biobank of colorectal cancer patients. Cell 161: 933-945, 2015.

25. DeRose YS, Wang G, Lin YC, Bernard PS, Buys SS, Ebbert MT, Factor R, Matsen C, Milash BA, Nelson E, et al: Tumor grafts derived from women with breast cancer authentically reflect tumor pathology, growth, metastasis and disease outcomes. Nat Med 17: 1514-1520, 2011.

26. Gould SE, Junttila MR and de Sauvage FJ: Translational value of mouse models in oncology drug development. Nat Med 21: 431-439, 2015.

27. Cassidy JW, Caldas C and Bruna A: Maintaining tumor heterogeneity in patient-derived tumor xenografts. Cancer Res 75 : 2963-2968, 2015.

28. Eirew P, Steif A, Khattra J, Ha G, Yap D, Farahani H, Gelmon K, Chia S, Mar C, Wan A, et al: Dynamics of genomic clones in breast cancer patient xenografts at single-cell resolution. Nature 518: 422-426, 2015.

29. Wulkersdorfer B, Zeitlinger M and Schmid M: Pharmacokinetic aspects of vascular endothelial growth factor tyrosine kinase inhibitors. Clin Pharmacokinet: Jul 23, 2015 (Epub ahead of print).

30. Jørgensen JT: Clinical application of companion diagnostics. Trends Mol Med 21: 405-407, 2015.

31. Sohal DP, Rini BI, Khorana AA, Dreicer R, Abraham J, Procop GW, Saunthararajah Y, Pennell NA, Stevenson JP, Pelley R, et al: Prospective clinical study of precision oncology in solid tumors. J Natl Cancer Inst 108: djv332, 2015.

32. André F, Bachelot T, Commo F, Campone M, Arnedos M, Dieras V, Lacroix-Triki M, Lacroix L, Cohen P, Gentien D, et al: Comparative genomic hybridisation array and DNA sequencing to direct treatment of metastatic breast cancer: A multicentre, prospective trial (SAFIR01/UNICANCER). Lancet Oncol 15: 267-274, 2014.

33. Hidalgo M, Bruckheimer E, Rajeshkumar NV, Garrido-Laguna I, De Oliveira E, Rubio-Viqueira B, Strawn S, Wick MJ, Martell J and Sidransky D: A pilot clinical study of treatment guided by personalized tumorgrafts in patients with advanced cancer. Mol Cancer Ther 10: 1311-1316, 2011.

34. Gao H, Korn JM, Ferretti S, Monahan JE, Wang Y, Singh M, Zhang C, Schnell C, Yang G, Zhang Y, et al: High-throughput screening using patient-derived tumor xenografts to predict clinical trial drug response. Nat Med 21: 1318-1325, 2015.

35. Clohessy JG and Pandolfi PP: Mouse hospital and co-clinical trial project - from bench to bedside. Nat Rev Clin Oncol 12: 491-498, 2015.

36. Reyes DK and Pienta KJ: The biology and treatment of oligometastatic cancer. Oncotarget 6: 8491-8524, 2015.

37. Friedman AA, Letai A, Fisher DE and Flaherty KT: Precision medicine for cancer with next-generation functional diagnostics. Nat Rev Cancer 15: 747-756, 2015.

38. Majumder B,BaraneedharanU,Thiyagarajan S, Radhakrishnan P, Narasimhan H, Dhandapani M, Brijwani N, Pinto DD, Prasath A, Shanthappa BU, Thayakumar A, Surendran R, Babu GK, Shenoy AM, Kuriakose MA, Bergthold G, Horowitz P, Loda M, Beroukhim R, Agarwal S, Sengupta S, Sundaram M, Majumder PK: Predicting clinical response to anticancer drugs using an ex vivo platform that captures tumour heterogeneity. Nat Commun 6: 6169, 2015 